\title{
Erratum to: Feature ranking of type 1 diabetes susceptibility genes improves prediction of type 1 diabetes
}

\author{
Christiane Winkler • Jan Krumsiek • Florian Buettner • \\ Christof Angermüller • Eleni Z. Giannopoulou • \\ Fabian J. Theis • Anette-Gabriele Ziegler • Ezio Bonifacio
}

Published online: 6 November 2014

(C) Springer-Verlag Berlin Heidelberg 2014

\section{Erratum to: Diabetologia}

DOI 10.1007/s00125-014-3362-1

Unfortunately, the following sentence was omitted from the Funding section of this paper. 'This work was supported by iMed-the Helmholtz Initiative on Personalized Medicine.'

The online version of the original article can be found at http://dx.doi.org/ $10.1007 / \mathrm{s} 00125-014-3362-1$.

C. Winkler $\cdot$ E. Z. Giannopoulou $\cdot$ A.-G. Ziegler $(\bowtie)$

Institute of Diabetes Research, Helmholtz Zentrum München, and

Forschergruppe Diabetes, Klinikum rechts der Isar, Technische

Universität München, Ingolstaedter Landstr. 1, 85764 Neuherberg,

Germany

e-mail: anette-g.ziegler@helmholtz-muenchen.de

C. Winkler $\cdot$ E. Z. Giannopoulou $\cdot$ A.-G. Ziegler

Forschergruppe Diabetes e.V., Neuherberg, Germany

J. Krumsiek · F. Buettner $\cdot$ C. Angermüller $\cdot$ F. J. Theis

Institute of Computational Biology, Helmholtz Zentrum München,

Neuherberg, Germany

F. J. Theis

Department of Mathematics, Technische Universität München,

Garching, Germany

E. Bonifacio $(\square)$

Center for Regenerative Therapies - Dresden, Technische

Universität, Fetscherstrasse 105, 01307 Dresden, Germany

e-mail: ezio.bonifacio@crt-dresden.de

E. Bonifacio

Paul Langerhans Institute Dresden, German Center for Diabetes

Research (DZD), Dresden, Germany

E. Bonifacio

Institute of Diabetes and Obesity, Helmholtz Zentrum München,

Neuherberg, Germany 